FTUV/92-27

\title{
Pionic effects in deep inelastic scattering off nuclei *
}

\author{
P. González ** and V.Vento ${ }^{\ddagger}$ \\ Departament de Física Teòrica and I.F.I.C. \\ Centre Mixt Universitat de València - C.S.I.C. \\ E-46100 Burjassot (València), Spain.
}

\begin{abstract}
The structure functions calculated in the Chiral bag model reproduce quite well, after appropriate perturbative evolution to large energy scales, the experimental data. We use these results to interpret the structure of the $E M C$ data as a quenching of the pion decay constant due to the in medium behavior of the nucleon. This explanation supports recent proposals of this phenomenon whose origin is the scale invariance of the $Q C D$ lagrangian.
\end{abstract}

*Supported in part by CICYT grant \# AEN90-0040 and DGICYT grant \# PB88-0064.

** Gonzalep@evalvx.bitnet

$\ddagger$ Vento@vm.ci.uv.es 


\section{Introduction}

The European Muon Collaborations $(E M C, N M C, S M C)$ have been shaking the foundations of our knowledge of the nucleon structure for the past ten years [1, 2]. In this paper we shall reanalyze the data of the original $E M C$ effect and find room for an heterodox explanation associated with some recent proposal about the scaling properties of coupling constants in a hadronic medium [3].

In order to explain the $E M C$ data, two broad classes of models have been developed [1]. One of the approaches comprises conventional nuclear physics models and dynamics. The contributions of all scattering components of the nucleus are taken into account, so in addition to the traditional protons and neutrons, constituent mesons and nucleon resonances have been considered [5]. Furthermore the properties of the nuclear medium, i.e. binding energy and density, can be incorporated [6].

A second approach is based on the so called $Q C D$ rescaling models. Here it is assumed that the internal $Q C D$ dynamics is modified in the nuclear environment leading to changes in the dynamics which can be described by associating a different renormalization group scale to each nuclei [7]. This mechanism interpreted in terms of a bag model has led to the much discussed phenomenon of nucleon swelling.

We pursue in this letter a different philosophy, namely we would like to establish a link, using experimental high energy data, between effective low energy models and asymptotic properties of the theory. This approach, originally proposed by Jaffe and Ross [8], has been followed by other groups as well [9]. In this spirit we have performed calculations of the structure functions in the Chiral bag model for single nucleon properties and extended them to incorporate medium effects.

\section{The structure function of the nucleon}

The first step in our approach is the calculation of the structure functions in a conventional low energy model. We have chosen for this purpose the chiral bag model, a model described in terms of quarks and gluons fields in the interior of a cavity, the bag, and in terms of pion fields in its exterior, while their coupling is defined to implement the symmetries of the strong interactions, color gauge symmetry and spontaneously broken chiral symmetry 10 . Following conventional techniques we calculate Compton scattering in the model by using the chiral expansion to first order in $\frac{1}{4 \pi f_{\pi}^{2} R^{2}}$, the effective expansion parameter. We obtain three types of terms : i) the photon-quark (antiquark) scattering inside the cavity, the only contribution appearing in the original MIT bag model calculation [11]; ii) the photon-quark (antiquark) scattering with pionic final state interactions, arising automatically in the chiral expansion to this order; iii) the photon-(virtual)pion interaction, corresponding to the meson exchange current contributions in the model. Only in this latter type of diagrams the photon scrutinizes, due to its short wave length, the structure of the boson. In order to connect with the asymptotic regime (only quarks, antiquarks and gluons) we incorporate, only in the meson exchange contribution, the structure of the pion [12]. In this way the model differs 
Figure 1: The isoscalar structure function of the nucleon. The solid line represents the structure function calculated in the Chiral bag model for $R=1 \mathrm{fm}, f_{\pi}=95 \mathrm{MeV}$. The dashed line represents its evolved from $\frac{Q_{0}}{\Lambda_{Q C D}}=1.5$ to $\frac{Q}{\Lambda_{Q C D}}=25$. Finally the vertical lines represent an eye guide to data points of the different experiments and their errors [18].

from the more naive convolution models [5] by distinguishing among the pionic contributions those with different time scales [13, 14. In particular those diagrams leading to final state quark-gluon interactions where a quark from the exchanged pion interacts with a quark from the nucleon by means of gluon exchange, which could change our internal exchanged pion time-scale, do not exist in our two phase approach. Between two different hadrons only pions can be exchanged and the relevant contributions are incorporated in the pionic description of the final state interaction. Certainly the model is very naive describing the final state interactions, but we would like to stress that, within an effective chiral theory approach, ours is the only possible calculation to lowest order in the chiral expansion.

Following Jaffe and Ross [8], radiative corrections are considered by adscribing a low energy scale, $Q_{0}$, to the model calculation and evolving to high $Q$ using the renormalization group equations. This procedure is certainly justified for the twist two operators, which are the only ones we are interested in here.

There has been a lot of discussion about the so called support problem [15, 16]. It is certainly true that bag calculations have a difficult center of mass problem which leads to structure functions which are not limited to the interval $0 \leq x \leq 1$, where $x$ is the Bjorken variable. However in most calculations over $95 \%$ of the momentum is inside that interval, as is our case. The support problem cannot be solved exactly, except in the two dimensional case. Therefore many approximations have been developed based on the calculations of quark distribution functions followed by a Peierls-Yoccoz projection. Due to the relativistic character of bag model calculation, this procedure is not only approximate, but very hard to implement. Moreover technical difficulties force one to severely restrict the number of 
modes in the calculation of the cavity propagators. In our case, the Chiral bag model, where one has to take also into account the pionic degrees of freedom, its development is tedious, technically complex and the errors due to truncation not controlable. In ref.(\|14]) the projection procedure was followed, but the calculation of terms to which the pion propagator could contribute was eluded.

Since our calculation was planned to unveil new dynamical possibilities, namely the implication of scale invariance into effective low energy actions, we have chosen simplicity, where matters were difficult to control, i.e., we have used unprojected states. On the contrary, whenever the scheme was well defined, we have not avoided calculational effort and have aimed to the highest precission possible. For example, we have carried out a quite complete (up to 50 modes have been considered) cavity perturbation theory calculation in the chiral bag including all the pionic diagrams.

We have forced our structure function to have the proper support, by restricting $x$ to vary in the correct interval $([0,1])$. We have analyzed the stability of the solution by enlarging or reducing the support about these endpoints. The stability of our results after truncation give us some confidence in the qualitative behavior of the evolved structure function obtained.

We have used an ingenious method of evolution [17 to leading order which requires relatively few moments: from the tenth moment on results do not change more than $15 \%$ for $x>0.05$. The truncation of the support affects mostly the high moments. Recall that it is precisely the high moments which might create disturbances in the calculation due to the ill-behavior of the structure function beyond $x=1$. This reconstruction method is therefore greatly responsible for the stability of our solution. However one has to be extremely careful performing the calculation since rounding errors can destroy the reconstruction procedure altogether. The method requires the operation with very large numbers to obtain relatively small results. In our case, a careful optimization of the calculational sequence has allowed us to compute up to a maximum of 35 moments. Beyond this number we run into precission problems. The the method was generalized to a different support in order to check convergence.

In Fig.(傮) we show the Chiral bag model structure function and the one that results from our evolution process. The parameters of the model have been fixed in part to reproduce the experimental low energy data, without much effort at precission (bag radius $R=1 \mathrm{fm}$, $f_{\pi}=95 \mathrm{MeV}$, which lead to a mass for the nucleon of $M \approx 1000 \mathrm{MeV}$ ). To leading order the remaining parameter is $\frac{Q_{0}}{\Lambda_{Q C D}}$, which we have fitted to get the correct behavior of the structure function and the maximum possible value for the gluon content. Our value for it of 1.5 is consistent with that of other calculations [9]. From this ratio and an experimental $\Lambda_{Q C D}=0.2 \mathrm{GeV}$ the low energy scale becomes $Q_{0}=0.3 \mathrm{GeV}$. We have evolved up to $Q=$ $5 \mathrm{GeV}$, although one reaches a slowly varying plateau in the evolution from $Q=1.5 \mathrm{GeV}$ on. It is clear from the figure that we overestimate the low $x$ behaviour of the structure function. On the other hand the gluon content of the evolved solution is about $47 \%$ instead of the experimental one of $55 \%$.

These two features seem to indicate that we should add to our description hard gluons at the quark model level through the one gluon exchange mechanism $(O G E)$ [9, 19]. In this 
case one would have gluons at the low evolution scale, which would also imply an increase in the $\frac{Q_{0}}{\Lambda}$ ratio and as a consequence a shift of the momentum from the low $x$ to intermediate $x$ quarks, approaching a overall better fit to the data.

We have calculated, with the additional assumption of a symmetric sea, the Drell-Yan process leading to a fit of similar quality. The gluon distribution comes out comparable to other more phenomenological approaches [4], but the full merit here corresponds to the $Q C D$ evolution and not to the model.

\section{The structure function in a nuclear medium}

By using effective chiral Lagrangians with a suitable incorporation of the scaling property of $Q C D$, Brown and Rho [3] have established an in-medium scaling law one of whose most appealing expressions reads

$$
\frac{M^{*}}{M}=\sqrt{\frac{g_{A}^{*}}{g_{A}}} \frac{f_{\pi}^{*}}{f_{\pi}}
$$

where $M^{*}, g_{A}^{*}$ and $f_{\pi}^{*}$ are the effective in-medium nucleon mass, axial coupling constant and pion decay constant respectively. The ratio of pion decay constants, our scaling parameter, will be labeled hereafter $\sigma$. We follow their prescription and assume that the in-medium effects can be incorporated by taking the effective Lagrangian with scaled couplings to calculate physical observables according to a chiral perturbation scheme. In our case this reduces to a chiral bag model picture in which the quarks, gluons and pions carry effective charges.

In our scheme, to lowest order in the chiral expansion, $g_{A}$ does not change in the medium, i.e.,

$$
g_{A}=\frac{5}{9} \frac{\varepsilon}{\varepsilon-1}
$$

where $\varepsilon=2.04 \ldots$ is the lowest mode of the cavity, which does not change with density.

This is not of major concern since experimentally $\sqrt{\frac{g_{A}^{*}}{g_{A}}}>0.9$ and quite close to one for intermediate nuclei, which are the ones used in EMC data. Moreover in our calculation the structure function is strictly independent on the mass as long as we adhere to the chiral perturbative scheme to lowest order (It turns out to be only a function of $\varepsilon$ and $f_{\pi} R$ ). Our scheme will produce a dependence of the structure function on $M R$ and of $g_{A}$ on $M R$ and $f_{\pi} R$ in higher orders in the chiral expansion [20]. We shall not consider these effects in the present work and hence the only effective medium dependent parameter to be considered is $f_{\pi}$.

In Fig.(2) we plot the ratio $\frac{F_{2}\left(f_{\pi}^{*}\right)}{F_{2}\left(f_{\pi}\right)}$ and compare it with the $E M C$ data. Certainly we are aware of Fermi motion corrections [4, 6], but to clarify our discussion we have not included them in our calculation.

Our aim has not been to obtain a one parameter $(\sigma)$ fit to the experimental EMC data, although from the results shown in Fig.(2) one could attempt to do so incorporating the missing ingredients (support corrections, loop contribution, fermi motion contributions, etc...). 
Figure 2: Ratio of structure functions for different values of the scaling parameter. The continuous curve corresponds to $\sigma=.95$ and the dashed curve to $\sigma=0.9$. The vertical lines give a schematic idea of the spread of data points of the $E M C$ effect for the different experiments and their errors [18].

We want to signal however, that the proposed mechanism is quite efficient in shifting momentum from the intermediate $x$ region to the low $x$ region, and that the data seem to support such a quenching of the effective parameters.

From our calculation we have also obtained the gluon distribution. Our mechanism does produce a change of it in going from free nucleons to in-medium nucleons, which is similar in shape to the structure function ratio, but smaller in magnitude. As in the free case, since gluons are produced solely via bremsstrahlung, the result is very much dependent on the evolution ratio.

Before finishing this section one caveat is necessary. The reader might find surprising that our data does not extend into the shadowing region. As is well known, shadowing has to do with the hadronic structure of the photon, and we have never aimed at discussing this physics. Therefore the very low $x$ data could only confuse the issue.

\section{Conclusion}

We have performed a calculation of the structure function of the nucleon using as boundary conditions of the renormalization group equations the structure functions calculated in the Chiral bag model. A qualitative fit to the experimental data is obtained with reasonable values of the parameters. In particular small value of the low energy scale parameter $Q_{0}$ may have to do with the omission of perturbative gluonic contributions to the structure function, relevant at this scale where the $Q C D$ coupling constant is sizeable. The smallness of $Q_{0}$ leads to an overestimate of the structure function for low $x$, since the evolution equations in 
this case shift too much quark momentum from the high to the low $x$ region.

Using the scaling argument of Brown and Rho, we have been able to obtain the inmedium structure functions. We see that the experimental results are compatible with such a mechanism. The quenching of $f_{\pi}$ is relatively small, as corresponds to low densities. Moreover once Fermi motion corrections are incorporated the data can be well fitted. Our aim, however is not to obtain a one parameter fit to the data, but to reveal that the scaling hypothesis could be confirmed in this scenario.

Peculiar features of the calculation are the rise for low $x$ and high $x$ of the ratio as can be seen in Fig.(2). The behavior at low $x$ is in our opinion a general characteristic of pionic contributions and will appear in any model calculation including them. It arises from the meson exchange diagram and is a direct consequence of the structure of the pion [12]. Certainly this rise at low x carries, due to momentum conservation a depletion of the intermediate $x$ region. On the contrary the rise at high $x$ is a peculiarity of the model. The confinement condition in the Chiral bag model leads to boundary conditions originating a quark pion interaction which becomes stronger as we decrease $f_{\pi}$. This interaction determines the strenght and $x$-dependence of the final state interaction terms (those of type ii)) producing for the scaled parameter an inverse shift pushing quarks from intermediate $x$ to high $x$ [12].

We must end by stating that in our opinion despite the technical difficulties that remain to be solved the approach used in this letter to signal a possible scaling scenario deserves to be further developed. The behavior of the structure function in Fig.(11), together with the results of other calculations [9, 14], makes the whole process appealing. Moreover we see that one can implement a low energy microscopic description by using high energy data, as long as one accepts the evolution assumption [8].

\section{Acknowledgement}

We acknowledge useful conversations with Marco Traini, José Peñarrocha and V. Sanjosé. P.G. is thankful to Celia for assistance in the optimization of the computer codes.

\section{References}

[1] J.J. Aubert, Phys. Lett. 123B (1983) 275.

[2] J. Ashman et al., Phys. Lett. B206 (1988) 364; P. Amaudruz et al., Phys. Rev. Lett. 66 (1991) 2712.

[3] G.E. Brown and M. Rho, Phys. Rev. Lett. 66 (1991) 2720.

[4] R.G. Roberts in The structure of the proton (Cambridge University Press 1990); R.D. Field in Applications of perturbative QCD (Addison Wesley Pub. Co. 1989; V. Barone and E. Predazzi, Ann. Phys. (Fr.) 12 (1987) 525. 
[5] C.H. Llewelyn Smith, Phys. Lett. 128B (1983) 107; M. Ericson and A.W. Thomas, Phys. Lett. 128B (1983) 112; J. Szwed, Phys. Lett 128B (1983) 245.

[6] C.A. Garcia Canal, E.M. Santangelo and H. Vucetich, Phys. Rev. Lett. 53 (1984) 1430; Phys. Rev. D35 (1986) 382.

[7] R.L. Jaffe, F.E. Close, R.G. Roberts and G.G. Ross, Phys. Lett. 134B (1984) 449; Phys. Rev. D31 (1985) 1004.

[8] R.L. Jaffe and G.G. Ross, Phys. Lett 93B (1980) 313.

[9] A.W. Schreiber, A.I. Signal and A.W. Thomas, Phys. Rev. D44 (1991) 2653; L. Conci and M. Traini, Few-Body Systems 8 (1990) 123; M. Traini, L. Conci and U. Moschella, Nucl. Phys. A544 (1992) 731; M. Traini, L. Conci and M. Melchiori, University of Trento UTF 237-1991; V. Barone and A. Drago, University of Ferrara 1992.

[10] V. Vento, M. Rho, E. Nyman, J.H. Jun, and G.E. Brown, Nucl. Phys. A470 (1980) 413.

[11] R.L. Jaffe, Phys. Rev. D11 (1975) 1953.

[12] V. Sanjosé, V. Vento and S. Noguera, Nucl. Phys. A470 (1987) 510; V. Sanjosé and V. Vento, Nucl. Phys. A501 (1989) 672.

[13] R.L.Jaffe, Comments in Nucl. Part. Phys. 13 (1984) 39.

[14] A.W. Schreiber, P.J. Mulders, A.I. Signal and A.W. Thomas, Phys. Rev. D45 (1992) 3069 .

[15] R.L. Jaffe, Ann. Phys. (N.Y.) 132 (1981) 32.

[16] A.I. Signal and A.W. Thomas, Phys. Rev. D40 (1989) 2832.

[17] F.J. Ynduráin, Phys. Lett. B74 (1978) 68; A. González-Arroyo, C. López and F.J. Ynduráin, Nucl. Phys. B153 (1979) 161; ibid B159 (1979) 512; ibid B166 (1980) 429.

[18] J.J. Hernández et al., Phys. Lett. B239 (1990) 1; R.G. Roberts and M.R. Whalley, J. Phys. G 17 (1991) D1.

[19] F. Myhrer and A.W. Thomas, Phys. Rev. D38 (1988) 1633.

[20] M. Rho, Phys. Rev. Lett. 54 (1985) 767; G. E. Brown and M. Rho, Phys. Lett. B 222 (1989) 324. 